\title{
Identificación y susceptibilidad de Candida spp. en el área ginecológica
}

\author{
Identification and susceptibility in Candida spp. in the gynecological area \\ Identificação e suscetibilidade em Candida spp. na área ginecológica
}

\author{
Julia María Orellana Quito \\ julyorellanaq@gmail.com \\ https://orcid.org/0000-0001-9052-4980
}

\author{
Karla Estefanía Pacheco Cárdenas \\ karla.pacheco@ucacue.edu.ec \\ https://orcid.org/0000-0002-9571-9389
}

\section{Programa de Maestría en Diagnóstico de Laboratorio Clínico y Molecular, Universidad Católica de Cuenca, Cuenca-Ecuador}

Recibido 26 de marzo 2021 | Arbitrado y aceptado 14 de abril 2021 | Publicado en 4 de mayo 2021

\section{RESUMEN}

La candidiasis es una infección causada por la acción patógena de Candida spp., catalogada como la segunda causa a nivel mundial de infección vaginal en mujeres con edades comprendidas entre 15 y 45 años. Siendo Candida albicans la más prevalente con distribución global. Uno de los problemas de importancia clínica es la resistencia a los antifúngicos debido al uso irracional de los mismos como terapia empírica y profilaxis. Objetivo. Caracterizar la infección por Candida spp., según: perfiles de susceptibilidad y frecuencias de candidiasis vulvo - vaginal relacionada con el balance de contenido vaginal. Así como, la presencia de coinfecciones en mujeres de edad fértil en las ciudades Cuenca - Azogues, que asisten a consulta en el Hospital Monte Sinaí, periodo enero noviembre de 2020. Materiales y métodos. Estudio de tipo descriptivo, documental de corte transversal, la muestra estuvo conformada por 136 aislados clínicos, se utilizó un muestreo por cobertura total. Resultados. El grupo etario que prevaleció fue de $19-39$ años. Candida albicans fue la más prevalente en muestras de secreción vaginal con $92.6 \%$, seguido de Candida glabrata 6.6 $\%$ y Candida parapsilosis con 0.7 \%. Las tasas de resistencia se diferenciaron en Miconazol con 19.9 \%, Itraconazol 16.9 \%, y Fluconazol 14\%. Conclusiones. El agente etiológico causal de candidiasis vulvo - vaginal fue Candida albicans. La interpretación del balance de contenido vaginal y perfiles de susceptibilidad, son herramientas clave para el óptimo tratamiento de la paciente con infecciones vaginales, evitando la automedicación y la farmacorresistencia.

Palabras clave. Candida; candidosis; vulvovaginitis; antifúngicos, susceptibilidad; farmacorresistencia

\begin{abstract}
Candidiasis is an infection caused by the pathogenic action of Candida spp., Classified as the second worldwide cause of vaginal infection in women aged between 15 and 45 years. Being Candida albicans the most prevalent with global distribution. One of the clinically important problems is resistance to antifungals due to their irrational use as empirical therapy and prophylaxis. Objective: To characterize the infection by Candida spp., according to: susceptibility profiles and frequencies of vulvo-vaginal candidiasis related to the balance of vaginal content. As well as, the presence of co-infections in women of childbearing age in Cuenca - Azogues cities, who attend a consultation at the Monte Sinaí Hospital, January - November 2020 period. Materials and methods. A descriptive, observational cross-sectional study, the sample consisted of 136 clinical isolates, a full coverage sampling was used. Results. The age group that prevailed was $19-39$ years. Candida albicans was the most prevalent in vaginal discharge samples with $92.6 \%$, followed by Candida glabrata $6.6 \%$ and Candida parapsilosis with $0.7 \%$. Resistance rates differed in Miconazole with $19.9 \%$, Itraconazole $16.9 \%$, and Fluconazole $14 \%$. Conclusions. The causative etiological agent of vulvo-vaginal candidiasis was Candida albicans. The interpretation of the balance of vaginal content and susceptibility profiles are a key tool for the optimal treatment of the patient with vaginal infections, avoiding self-medication and drug resistance.
\end{abstract}

J0: Coordinadora del departamento de Microbiología del Hospital Monte Sinaí. Diplomado internacional en Infectología. Universidad de los Hemisferios. Certificación internacional en Higiene y desinfección Hospitalaria. Universidad Central de Quito - Universidad Española de Seguridad. Universidad Católica de Cuenca, Ecuador.

KP: Química farmaceuta, Universidad Católica de Cuenca. Master en Bacteriología y Micología, Universidad de la Habana. Docente de la carrera de Biofarmacia, Bioquímica y farmacia, Universidad Católica de Cuenca. Encargada del Departamento de de Cuenca. Encargada del Departamento de Titulación de Biofarmacia, Bioquímica y Ecuador.

Key words: Candida; candidosis; vulvovaginitis; drug resistance; susceptibility 
J0: Coordinadora del departamento de Microbiología del Hospital Monte Sinaí. Diplomado internacional en Infectología Universidad de los Hemisferios. Certificación internacional en Higiene y Certificación internacional en Higiene desinfección Hospitalaria. Universidad Central de Quito - Universidad Española de Seguridad. Universidad Católica de Cuenca, Ecuador

KP: Química farmaceuta Universidad Católica de Cuenca. Master en Bacteriologí Católica Cuenca. Master en Bacteriologra y Micología, Universidad de la Habana. Docente de la carrera de Biofarmacia Bioquímica y farmacia, Universidad Católica de Cuenca. Encargada del Departamento de Titulación de Biofarmacia, Bioquímica y Farmacia. Universidad Católica de Cuenca, Ecuador.

\section{RESUMO}

Candidíase é uma infecção causada pela ação patogênica de Candida spp., Classificada como a segunda causa mundial de infecção vaginal em mulheres com idade entre 15 e 45 anos. Sendo Candida albicans a mais prevalente com distribuição global. Um dos problemas clinicamente importantes é a resistência aos antifúngicos devido ao seu uso irracional como terapia empírica e profilaxia. Objetivo. Caracterizar a infecção por Candida spp., De acordo com: perfis de suscetibilidade e frequências de candidíase vulvovaginal relacionadas ao equilíbrio do conteúdo vaginal. Assim como, a presença de coinfecções em mulheres em idade fértil nos municípios de Cuenca - Azogues, atendidas em consulta no Hospital Monte Sinaí, período janeiro - novembro de 2020. Materiais e métodos. Estudo descritivo, observacional, transversal, a amostra foi composta por 136 isolados clínicos, foi utilizada uma amostra de cobertura total. Resultados. A faixa etária prevalente foi de 19 a 39 anos. Candida albicans foi a mais prevalente nas amostras de corrimento vaginal com 92,6\%, seguida por Candida glabrata 6,6\% e Candida parapsilosis com 0,7\%. As taxas de resistência diferiram no Miconazol com 19,9\%, Itraconazol 16,9\% e Fluconazol 14\%. Conclusões. 0 agente etiológico causador da candidíase vulvovaginal foi Candida albicans. A interpretação do equilíbrio do conteúdo vaginal e dos perfis de suscetibilidade é uma ferramenta fundamental para o tratamento ideal da paciente com infecções vaginais, evitando a automedicação e a resistência aos medicamentos

Palavras-chave: Candida; candidose; vulvovaginite; antifúngicos, suscetibilidade; resistência a droga

\section{INTRODUCCIÓN}

$\mathrm{L}$ a candidiasis vulvo-vaginal (CVV), es una infección micótica común ocasionada por el sobrecrecimiento del agente fúngico Candida spp., como patógeno oportunista (1). La distribución geográfica de la candidosis es universal, entre un $85-95 \%$ de los aislamientos corresponden a Candida albicans y el porcentaje restante pertenecen generalmente a Candida glabrata (2), esta última es más frecuente en mujeres diabéticas (3). La CVV se presenta con amplia diversidad de cuadros clínicos, asociados a: estado inmunológico del paciente, trastornos de la glucorregulación, diabetes mellitus, obesidad, embarazo, estrés, contracepción hormonal, entre otras $(4,5)$.

La Candida spp., forma parte de la microbiota vaginal, no obstante, cuando existe desequilibrio ocasionado por cambios a nivel del pH ácido vaginal o desbalance hormonal (6), se favorece su establecimiento y reproducción. A raíz de lo cual, se presenta la sintomatología asociada como: prurito, ardor, dolor, descarga vaginal anormal, dispareunia, edema vaginal y eritema vulvar (7).

La CVV es la segunda causa de infección vaginal a nivel mundial, que afecta principalmente a mujeres en edades fértiles entre los 15-45 años y en el tercer trimestre de gestación. Como consecuencia se duplica la prevalencia y recurrencia de candidosis por los elevados niveles de estrógenos y glucocorticoides (8), lo cual provoca disminución de los mecanismos de defensa vaginal frente a gérmenes oportunistas (9).

Es significativo mencionar que, en las mujeres con vida sexual activa el $75 \%$ adquieren un episodio de $\mathrm{CVV}$ en su vida. De las cuales un 45 a $50 \%$ desarrollará dos o más episodios, un 10 a $20 \%$ presentará complicaciones y el $5 \%$ padece de forma recurrente más de cuatro CVV al año (4). 
La vulvovaginitis por Candida en Estados Unidos de América (EEUU), constituyen una de las principales causas de asesoramiento ginecológico por la presencia de signos y síntomas vaginales, con reportes de más de 10 millones de consultas al año (10).

En México, es considerada como la única micosis de reporte obligatorio ante las autoridades sanitarias, a más de ello se exhibe que CVV en Latinoamérica, es muy similar a la reportada en países como EEUU; evidentemente $C$. albicans, prevalece como el principal agente etiológico (11).

El Ministerio de Salud Pública del Ecuador menciona que la colonización vaginal por Candida es frecuente entre mujeres atendidas con infecciones de transmisión sexual (ITS), extendiéndose muchas de las veces a la zona ano-rectal. En este sentido, en Ecuador las infecciones cérvico-vaginales, abarcan más de un tercio de la consulta ginecológica y su frecuencia parece estar en aumento (12). Es difícil determinar la incidencia real de esta patología debido a que, también se presenta de forma asintomática. Sin embargo, en el 2017 en un estudio realizado en la Provincia del Azuay en Cuenca se determinó que la prevalencia de CVV fue de 30,33\% (13).

A pesar de que, la Candida albicans es el agente causal de candidosis vaginal en el $80-90 \%$ de los casos (9), la importancia prevalece en el incremento de otras especies asociadas a CVV, aunado a la creciente resistencia a diversos agentes antifúngicos (14). En los últimos años se ha incrementado la proporción de infecciones atribuidas a otros miembros del género. Entre estos destaca C. glabrata, considerada en la última década como patógeno emergente tras aislarse de pacientes con vaginitis, cuya preocupación se asocia por la disminución de sensibilidad a fluconazol que puede ser de 4 a 32 veces menos sensibles que $C$. albicans (15).

En la actualidad los estándares para la determinación de resistencia antifúngica, se basan en los lineamientos establecidos por: "Clinical and Laboratory Standards Institute" (CLSI) y el "European Committee on Antimicrobial Susceptibility Testing" (EUCAST) (16). Estas instituciones brindan protocolos para optimizar los métodos de detección de resistencia, especialmente a los azoles, permitiendo mejorar la eficacia de la terapia antifúngica (17).

Por todo lo anterior, resulta relevante una identificación idónea, así como, sugerir una opción de tratamiento válida debido a que constituyen un dato de importancia clínica (18).

El objetivo de este estudio fue caracterizar la infección por Candida spp., según: perfiles de susceptibilidad $y$ frecuencias de candidiasis vulvovaginal relacionada con el balance de contenido vaginal. Así como, la presencia de coinfecciones en mujeres de edad fértil en las ciudades Cuenca-Azogues, que asisten a consulta en el Hospital Monte Sinaí, durante el periodo enero-noviembre de 2020.

\section{MATERIALES Y MÉTODOS}

$\mathrm{E}$ l presente estudio tiene un enfoque cuantitativo, de tipo descriptivo, documental de corte transversal. La población estuvo conformada por los informes de 136 aislados clínicos que fue la totalidad de mujeres en edad fértil comprendidas entre 15 y 45 años de edad, que asistieron a la consulta de ginecología (muestreo por cobertura total). Los datos fueron recopilados de la fuente secundaria del departamento de microbiología del 
Hospital Monte Sinaí en las ciudades de Cuenca y Azogues en el período eneronoviembre 2020.

Los criterios de inclusión que se consideraron para el estudio fueron el registro de pacientes en edad fértil comprendida entre 15-15 años y el registro de pacientes procedentes de Cuenca y Azogues. El criterio de exclusión establecido fueron los registros de aislados clínicos que no tengan información completa o fuera del periodo de recolección.

\section{Procesamiento, análisis, resumen y presentación de la información}

De la base de datos se recolectó la información basada en las siguientes variables: (a) edad cronológica. (b) ciudad de procedencia. (c) perfiles de susceptibilidad. (d) balance de contenido vaginal. (e) coinfección.

Para el análisis estadístico se generó una base de datos en el programa IMB SPSS (versión 20.0). Se realizó una estadística descriptiva, análisis de frecuencia y chicuadrado. Para la presentación de los resultados se empleó tablas de simple y doble entrada y gráficos mediante el empleo de diagramas de barras.

\section{Aspectos éticos}

Esta investigación se fundamentó según los principios éticos establecidos en la Declaración de Helsinki, "Adendum de Taiwán", la cual señala la protección y privacidad a los datos de los registros primarios de pacientes de los cuales se obtuvieron los aislados de Candida spp., objetos de estudio, mismos que se manejaron con estricta confidencialidad, sin vulnerar el derecho de ningún paciente, debido a que se resguardó la base de datos, los nombres de pacientes fueron codificados en clave, dicha información no se utilizará con otros fines, no tendrán manejo público y no se harán nuevas investigaciones, respetando para lo que fueron tomadas, que fue el diagnóstico clínico.

El protocolo de la investigación fue evaluado y aprobado por los directivos y jefatura del Laboratorio de Microbiología del Hospital Monte Sinaí de la ciudad de Cuenca en Ecuador para la utilización de los registros ingresados en su base de datos del periodo comprendido entre enero-noviembre 2020.

\section{RESULTADOS}

$\mathrm{E}$ l total de muestras analizadas durante el periodo enero noviembre de 2020, fueron 136 , con frecuencia elevada en mujeres de edades comprendidas entre 19 - 39 años (73.5\%) seguidas de los grupos etarios comprendidos entre 40 - 45 años (22.8\%) y de 15 a 18 años (3.7\%).

$\mathrm{Al}$ analizar la especie de Candida de mayor prevalencia se aprecia que del $100 \% \quad(n=136)$ de las muestras en estudio, el $92,6 \%$ se correspondió con $C$. albicans, el 6,6\% C. glabrata y finalmente el 0,7\% C. parapsilosis. Tabla 1. 
Tabla 1. Distribución de Candida según especie

\begin{tabular}{clrrrr}
\hline & Frecuencia & Porcentaje & $\begin{array}{c}\text { Porcentaje } \\
\text { válido }\end{array}$ & $\begin{array}{r}\text { Porcentaje } \\
\text { acumulado }\end{array}$ \\
\hline \multirow{4}{*}{ Válidos } & Candida albicans & 126 & 92,6 & 92,6 & 92,6 \\
& Candida glabrata & 9 & 6,6 & 6,6 & 99,3 \\
& Candida & 1 &, 7 &, 7 & 100,0 \\
& parapsilosis & & & & \\
\hline & \multicolumn{1}{c}{ Total } & $\mathbf{1 3 6}$ & $\mathbf{1 0 0 , 0}$ & $\mathbf{1 0 0 , 0}$ & \\
\hline
\end{tabular}

En función de conocer la susceptibilidad, se determinó que frente a los antifúngicos testeados, el que presentó mayor tasa de resistencia fue miconazol con $19.9 \%$, itraconazol con $16.9 \%$ y fluconazol con $14 \%$. (Tabla 2).

Tabla 2. Especies de Candida y perfil de susceptibilidad.

\begin{tabular}{|c|c|c|c|c|c|}
\hline \multirow{2}{*}{\multicolumn{2}{|c|}{ PERFIL DE SUSCEPTIBILIDAD }} & \multicolumn{4}{|c|}{ ESPECIES DE CANDIDA } \\
\hline & & \multirow{2}{*}{$\begin{array}{r}\begin{array}{c}\text { Candida } \\
\text { albicans } \\
\text { Recuento }\end{array} \\
103\end{array}$} & \multirow{2}{*}{$\begin{array}{r}\begin{array}{c}\text { Candida } \\
\text { glabrata } \\
\text { Recuento }\end{array} \\
8\end{array}$} & \multirow{2}{*}{$\begin{array}{c}\text { Candida } \\
\text { parapsilosis } \\
\text { Recuento }\end{array}$} & \\
\hline \multirow{3}{*}{ FLUCONAZOL } & SENSIBLE & & & & 1 \\
\hline & INTERMEDIO & 5 & 0 & & 0 \\
\hline & RESISTENTE & 18 & 1 & & 0 \\
\hline \multirow{3}{*}{ ITRACONAZOL } & SENSIBLE & 102 & 7 & & 1 \\
\hline & INTERMEDIO & 3 & 0 & & 0 \\
\hline & RESISTENTE & 21 & 2 & & 0 \\
\hline \multirow{3}{*}{ KETOCONAZOL } & SENSIBLE & 114 & 8 & & 0 \\
\hline & INTERMEDIO & 4 & 0 & & 0 \\
\hline & RESISTENTE & 8 & 1 & & 1 \\
\hline \multirow{3}{*}{ MICONAZOL } & SENSIBLE & 100 & 7 & & 1 \\
\hline & INTERMEDIO & 1 & 0 & & 0 \\
\hline & RESISTENTE & 25 & 2 & & 0 \\
\hline \multirow{3}{*}{ VORICONAZOL } & SENSIBLE & 119 & 9 & & 1 \\
\hline & INTERMEDIO & 0 & 0 & & 0 \\
\hline & RESISTENTE & 7 & 0 & & 0 \\
\hline \multirow{3}{*}{$\begin{array}{c}\text { ANFOTERICINA } \\
\text { B }\end{array}$} & SENSIBLE & 125 & 9 & & 1 \\
\hline & INTERMEDIO & 0 & 0 & & 0 \\
\hline & RESISTENTE & 1 & 0 & & 0 \\
\hline
\end{tabular}

El análisis para identificar la prevalencia de coinfecciones en los diferentes grupos etarios, se presentan en el Gráfico 1, que permiten determinar que, las coinfecciones se presentaron con mayor frecuencia en el grupo etario comprendido ente 19 - 39 años de edad, con predominio de Gardnerella vaginalis y Streptococcus agalactiae. 


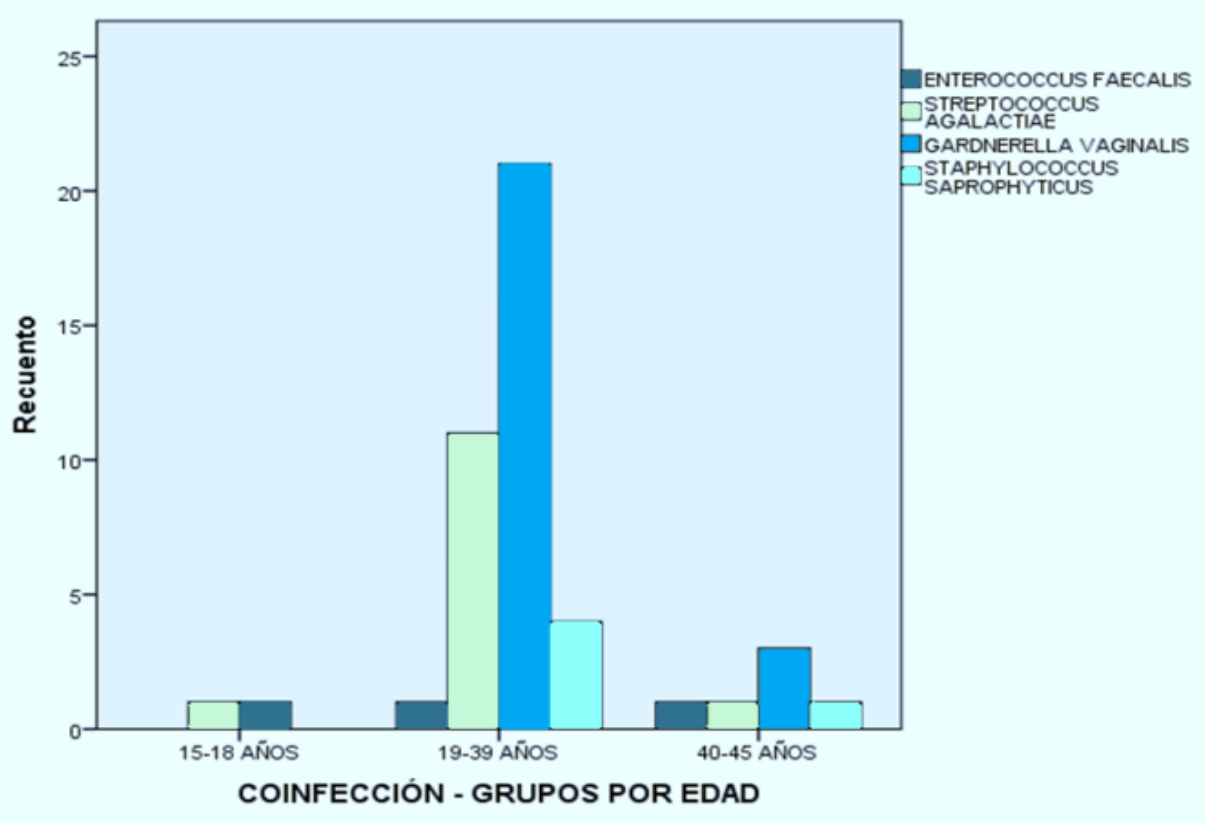

Gráfico 1. La coinfección asociada a grupo etario.

El Balance de Contenido Vaginal (BACOVA) se presenta acorde al desarrollo en los aislados obtenidos, diferenciándose claramente la ausencia de vaginosis, el desbalance de contenido vaginal y la vaginosis bacteriana, caracterizada principalmente por el desarrollo de Gardnerella vaginalis. Gráfico 2.

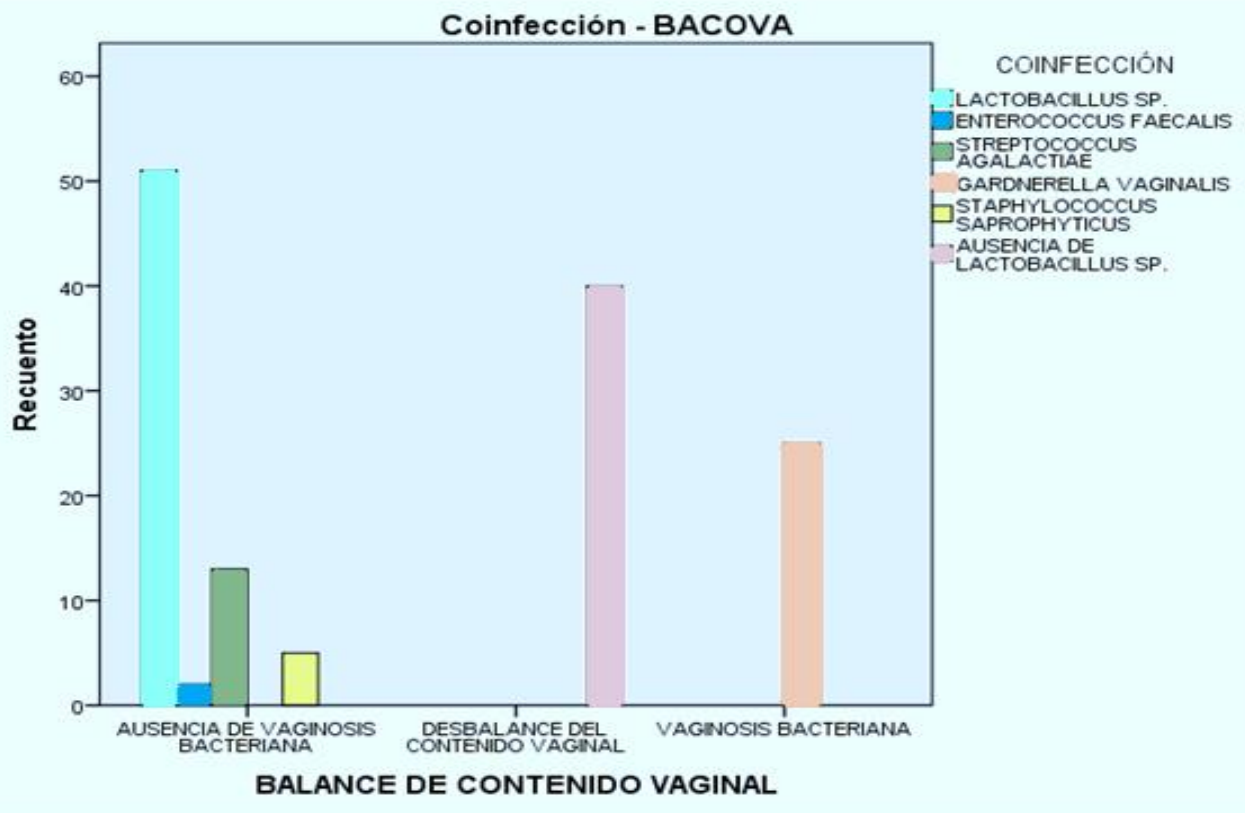

Gráfico 2. Relación del balance de contenido vaginal asociado a coinfección. 


\section{Discusión}

La CVV es la segunda causa en orden de frecuencia de vulvovaginitis en la mujer en edad fértil, se asocia a cambios hormonales durante el ciclo menstrual, anticonceptivos orales, diabetes, resistencia a la insulina, uso de antibióticos y prácticas sexuales poco habituales (19).

La colonización de Candida spp., en adolescentes y mujeres en edad sexual madura, se asocia a cambios en el nivel de $\mathrm{pH}$ vaginal y cambios hormonales, ocurridos en la fase lútea. Mientras que, en las mujeres posmenopáusicas se asocia a enfermedades de base, predominantemente diabetes mellitus en la que los niveles de glucosa altos influyen en la adherencia y fijación de las levaduras a la piel y las mucosas, lo cual favorece su proliferación e interfiere en la respuesta inmunitaria del huésped $(20,21)$.

Por el amplio rango etario al cual puede afectar, aunado a los factores de base, en la actualidad es considerada a nivel a mundial como un problema común de salud pública, se sugiere que la causa asociada es la falta de lineamientos en la confirmación microbiológica en pacientes con sospecha de CVV, lo cual impide un diagnóstico veraz que impide instaurar un tratamiento dirigido, promoviendo el uso irracional de antifúngicos y la sobrevenida de resistencia antifúngica (22).

C. albicans, es la especie más prevalente en infecciones vaginales micóticas como se denota en las investigaciones de Betancourt y Carrera (23) así como en el establecido por Aguilar G., et al (24), quienes identificaron a la C. albicans como el agente causal primordial en candidiasis vulvovaginal.

El fluconazol, demostró ser la opción terapéutica más eficaz en pacientes colonizadas por primera vez, como para pacientes con CVV recurrente, lo cual concuerda con el estudio realizado por
Quintero que demuestra la eficacia terapéutica que presenta frente a $C$. albicans mantenida en continuidad del tiempo, asociada al mecanismo que ejerce sobre la inhibición del ergosterol $(24,25)$.

Sin embargo, la susceptibilidad de miconazol se ve ensombrecida con un alto porcentaje de resistencia frente a $C$. albicans, contrario al estudio de Panizzo M., Pérez C., Maniscalchi M., que indican la eficacia terapéutica de miconazol en mujeres con infecciones primarias mientras que, en candidiasis vulvovaginal recurrente se determinó el incremento de resistencia a este antifúngico. La disminución en efectividad se debe a la prescripción irracional de antifúngicos como profilaxis y al incremento en dosis terapéuticas, lo cual puede provocar mutaciones moleculares en las enzimas de síntesis de ergosterol, así como promover la alteración de bombas de eflujo (26).

Los principales microorganismos causales de coinfección en CVV son Gardnerella vaginalis y Streptococcus agalactiae, miembros de la flora saprófita vaginal normal, son considerados como oportunistas, que, en disminución de Lactobacillus sp., pueden originar vaginitis e inflamación pélvica y parto o ruptura prematura de membranas respectivamente, agravando el cuadro clínico de la paciente $(17,27)$.

\section{CONCLUSIONES}

$\mathrm{E}$ 1 agente etiológico causal de candidiasis vulvo-vaginal fue la Candida albicans, la cual es reconocida mundialmente como un problema de salud pública. Se evidenció que afecta principalmente a pacientes en edad fértil, otro aspecto importante a considerar fue el incremento de resistencia 
antifúngica reflejado en el presente trabajo de investigación.

Lo expuesto anteriormente, refleja la importancia de caracterizar el agente causal de CVV. La interpretación del balance del contenido vaginal junto con los perfiles de susceptibilidad denota herramientas clave para el óptimo tratamiento de la paciente con infecciones vaginales, evitando la automedicación y por consiguiente la farmacorresistencia.

\section{REFERENCIAS BIBLIOGRÁFICAS}

1. Pineda J, Cortés A, Uribarren T, Castañón L. Candidosis vaginal. Revisión de la literatura y situación de México y otros países latinoamericanos. Rev. Méd. Risaralda [Internet]. 2017 [citado 7 de diciembre de 2020];23(1):38 - 44 . Disponible en: http://www.scielo.org.co/pdf/rmri/v23n1 /v23n1a09.pdf

2. Cires M, Freijoso E, Silva L, Cutié E, Ortega M, Sansó F, et al. Guía para la práctica clínica de las infecciones vaginales. Rev Cubana Farm [Internet]. 2003 [citado 22 de diciembre de 2020];37(1). Disponible en: http://scielo.sld.cu/scielo.php?script=sci_a rttext\&pid=S003475152003000100006\&lng=es.

3. Sanchéz E. Manejo de vulvovaginitis en la atención primaria. Rev Médica Sinerg [Internet]. 2018 [citado 15 de diciembre de 2020];3(8):1320. Disponible en: https://revistamedicasinergia.com/index. php/rms/article/view/305

4. Ramírez A, Pereiro M, Toribio J. Vulvovaginitis de repetición. Valoración diagnóstica y manejo terapéutico. Actas Dermosifiliogr. [Internet]. 2008 [citado 02 febrero de 2021];99(3):190-8. Disponible en: https://www.actasdermo.org/es-pdfS0001731008746556

5. Fernández M, Lombardía J. Vulvovaginitis y cervicitis en la práctica diaria. SEMERGEN [Internet]. 2002 [citado
01 de diciembre 2020];28(1):1520. Disponible en: https://dialnet.nirioja.es/servlet/articulo? codigo $=3788676$

6. Troyano J, Apuntes y reflexiones sobre la terapia local de la vulvovaginitis. Porg Obste [Internet]. 2019 [citado 22 de diciembre de 2020];62(3):205-215. Disponible en: https://sego.es/documentos/progresos/v 62-2019/n3/01_Editorial_6203-esp.pdf

7. Stockdale C, Boardman L. Diagnóstico y Tratamiento de Dermatosis Vulvares. Obstet Gynecol [Internet]. 2018 [citado 06 de enero de 2021];117-131. Disponible en: https://journals.lww.com/greenjournal/D ocuments/Feb2018_Translation_Stockdale .pdf

8. Benedí J, Sagrario M. Tratamiento de las infecciones ginecológicas. Revisión. Farmacia Profesional. 2009 [citado 11 de enero de 2021];23(1):52-7. Disponible en: http://www.scielo.org.co/pdf/rmri/v2 3n1/v23n1a09.pdf

9. Andrés P. Alteraciones vulvovaginales. ADOLESCERE [Internet]. 2019 [citado el 14 de diciembre de 2020];7(1):26-38. Disponible en: https://www.adolescenciasema.org/temade-revision-alteraciones-vulvovaginalesbartolinitis-leucorreas-traumatismosvaginosis-e-infecciones-de-transmisionsexual-p-andres-adolescere-2019-vii-1-2638/

10. Pineda J, Cortés A, Uribarren T, Castañón L. Candidosis vaginal: Revisión de la literatura y situación de México y otros países latinoamericanos. Rev. Méd. Risaralda [Internet]. 2017 [citado 10 de marzo de 2021];23(1):38-44. Disponible en: http://www.scielo.org.co/scielo.php?s cript=sci_arttext\&pid=S0122-

06672017000100009

11. Rivera $R$, Flores $R$, Arriaga $M$. Identificación de especies de Candida causantes de vaginitis en la población mexicana. Enferm Infecc Microbiol Clín [Internet]. 2006 [citado 18 de febrero de 2021];24(10):634-6. Disponible en: https://www.elsevier.es/es-revista- 
enfermedades-infecciosas-microbiologiaclinica-28-pdf-13095375

12. Ministerio de Salud Pública del Ecuador. Diagnóstico y tratamiento de la infección vaginal en obstetricia. Guía de Práctica Clínica. Ministerio de Salud Pública, Dirección Nacional de Normatización-MSP; 2014: 1-22. Disponible en: https://enlace.17d07.mspz9.gob.ec/bibliot eca/prov/guias/guias/Diagn\%C3\%B3stico $\% 20 \mathrm{y} \% 20$ tratamiento\%20de\%20la\%20in fecci\%C3\%B3n\%20vaginal $\% 20$ obst $\%$ C3\% A9trica.pdf

13. Sacoto G. Prevalencia de candidiasis vulvovaginal $\mathrm{y}$ factores asociados en pacientes del Centro Ambulatorio Central del Instituto Ecuatoriano de Seguridad Social, Loja 2014. [Tesis Doctoral]. Cuenca: Universidad de Cuenca; 2015. Recuperado a partir de: http://dspace.ucuenca.edu.ec/handle/123 $456789 / 21426$

14. Solís N. Determinación de la resistencia al fluconazol y nistatina mediante el fungigrama en vaginosis crónica causada por candida albicans en mujeres de 18-35 años que acuden a CEMOPLAF (CENTRO MÉDICO DE ORIENTACIÓN Y PLANIFICACIÓN FAMILIAR) LATACUNGA 2014. [Tesis]. Ambato: Universidad Técnica de Ambato; 2015. Recuperado a partir de http://repositorio.uta.edu.ec/handle/123 456789/8713

15. Perea E. Infecciones del aparato genital femenino: vaginitis, vaginosis y cervicitis. Med - Programa Form Médica Contin. Medicine [Internet]. 2018 [citado 19 de febrero de 2021];10(57):39104. Disponible

en: https:/clinicainfectologica2hnc.webs.fc m.unc.edu.ar/files/2018/03/Vaginitis_vag inosis_cervicitis_Medicine.pdf

16. Tapia P. Actualización en pruebas de susceptibilidad antifúngica. Rev Chil Infectol. [Internet]. 2016 [citado 18 de febrero de 2021]; 26(2):144-50. Disponible

en: https://www.researchgate.net/publica
tion/308906441_Mecanismos_de_resisten cia_antifungica_de_los_azoles_en_Candida_ albicans_Una_revision

17. López-Ávila K, Dzul-Rosado KR, LugoCaballero C, et al. Mecanismos de resistencia antifúngica de los azoles en Candida albicans. Una revisión. Rev Biomed [Internet]. 2016 [citado 21 de diciembre de 2020]; 27(3):127-136. Dispnible en: https://www.medigraphic.com/pdfs/revbi o/bio-2016/bio163e.pdf

18. Miró M, Rodríguez E, Vigezzi $C$, Icely $P$, Gonzaga de Freitas M, Riera F, et al. Candidiasis vulvovaginal: una antigua enfermedad con nuevos desafíos. Rev Iberoam Micol [Internet]. 2017 [citado 19 de diciembre de 2020];34(2):6571. Disponible en: https://www.sciencedirect.com/science/a rticle/abs/pii/S1130140617300281

19. Delmonte $M$, Fernández $P$, Robertiz $S$, González E, Arcaya N. Frecuencia del género Candida en vagina de mujeres en edad reproductiva. Kasmera [Internet]. 2017 [citado 1 de mayo de 2021];45(1):44-51. Disponible en: https://www.redalyc.org/jatsRepo/3730/ 373061522006/373061522006.pdf

20. López-Olmos J. Infecciones vaginales y lesiones celulares cervicales (II). Estacionalidad y relación con el ciclo menstrual. Clínica E Investig En Ginecol Obstet. [Internet].2012 [citado 1 de mayo de 2021];39(1):2-9. Disponible en:https://www.elsevier.es/es-revistaclinica-e-investigacion-ginecologiaobstetricia-7-pdf-S0210573X10001346

21. Lara J. Cepas de Candida albicans en pacientes con diabetes mellitus.Rev Cien Mund Inv. RECIMUNDO [Internet]. 2019 [citado 1 de mayo de 2021];3(1):13061339 Disponible en: https://recimundo.com/index.php/es/arti cle/view/418/615

22. Tapia C. Antifungal drug resistance: mechanisms, epidemiology, and consequences for treatment. Rev Chil Infectol. [Internet] 2012 [citado 1 de mayo de 2021];29(3):357-357. Disponible en: 
https://scielo.conicyt.cl/pdf/rci/v29n3/ar t20.pdf

23. Betancourt E, Carrera V. Prevalencia de candidiasis vaginal en mujeres embarazadas de Quito-Ecuador: identificación de especies utilizando dos medios de cultivo. Rev Med Vozandes [Internet]. 2012 [citado 1 de mayo de 2021];23: 113-118. Disponible en: https://docs.bvsalud.org/biblioref/2019/ 09/1021293/04_ao_03.pdf\#: :text=La\%2 0prevalencia\%20de $\% 20$ candidiasis $\% 20$ va ginal,\%25)\%20en\%20SBA\%20y\%20CAC

24. Aguilar G, Araujo P, Godoy E, Falcón $M$, Centurión MG, Ortiz $R$, et al. Identificación y características de Candida spp. en secreción vaginal de pacientes embarazadas y no embarazadas. Mem Inst Investig Cienc Salud [Internet]. 2017 [citado 5 de mayo de 2021];15(3):6-12. Disponible en: http://scielo.iics.una.py/scielo.php?script= sci_abstract\&pid=S1812-

95282017000300006\&lng=en\&nrm=iso\&t lng=es. Mem Inst Investig En Cienc Salud. diciembre de 2017;15(3):6-12.

25. Gómez C. Resistencia de levaduras del género Candida al fluconazol. Asc Col Inf. [Internet]. 2010 [citado 15 de enero de 2021];14(2):172-S180. Disponible en: http://www.scielo.org.co/pdf/inf/v14s2/ v14s2a09.pdf

26. Panizzo $M$, Pérez $C$, Maniscalchi $M$. Susceptibilidad in vitro a los antifúngicos de Candida sp. y serotipos de Candida albicans aisladas de pacientes con vaginitis primaria y resurrente. Rev Soc Venez Microbiol [Internet]. 2000 [citado 19 de febrero de 2021];20(1):01-01. Disponible en:

http://ve.scielo.org/scielo.php?script=sci arttext\&pid=S1315-25562000000100004

27. Ibarrola $\mathrm{M}$, Benito J, Azcona B, Zubeldía N. Patología infecciosa: vulvovaginitis, enfermedades de transmisión sexual, enfermedad inflamatoria pélvica, abscesos tuboováricos. An Sis Navar [Internet]. 2009 [citado 1 de mayo de 2021];32(1): 29-38. Disponible en: https://scielo.isciii.es/pdf/asisna/v32s1/g inecologia3.pdf

Conflicto de interese: Los autores declaran que no existe conflicto de intereses para la publicación del presente artículo.

Financiamiento: Autofinanciamiento Agradecimiento: No declaran 